\title{
Comprehensive Analysis of the Prognostic Value of ITGB Superfamily Members in Gastric Cancer Using Bioinformatics Analysis
}

\section{Peixin Lu}

Tongji University School of Medicine

Jinying Jiang

Shanghai East Hospital

Jun Lu (D 109303454@qq.com)

Shanghai East Hospital https://orcid.org/0000-0001-6612-6229

\section{Research}

Keywords: Gastric cancer, ITGB superfamily, Bioinformatics, Prognosis

Posted Date: April 23rd, 2021

DOI: https://doi.org/10.21203/rs.3.rs-435154/v1

License: (c) (i) This work is licensed under a Creative Commons Attribution 4.0 International License.

Read Full License 


\section{Abstract}

Background: Gastric cancer (GC) is one of the most common malignant tumors worldwide with a high incidence and mortality, making it urgent to search for potential biomarkers to improve the prognosis of patients. Integrin $\beta$ (ITGB) superfamily members have been reported to participate in the biological process in various tumors. However, the expression and prognostic value of ITGBs in GC have not been clarified. In this study, the expression and roles of ITGBs in stomach adenocarcinoma (STAD) were investigated with ONCOMINE, UALCAN, The Human Protein Atlas, GEPIA, Kaplan-Meier Plotter, NetworkAnalyst, cBioPortal, STRING, GeneMANIA, David 6.8 and TIMER.

Results: The transcriptional levels of ITGB1, 2, 4, 5, 6 and 8, and the protein expression of ITGB4, 5, 6 and 8 were significantly elevated while the transcriptional level of ITGB7 was significantly reduced in GC. High mRNA expression of ITGB1, 3 and 5, and low mRNA expression of ITGB4 were associated with short overall survival (OS), and high mRNA expression of ITGB6 was associated with short disease-free survival (DFS) in STAD patients. We also identified DAB2 as the potential transcription factor for ITGB3 and ITGB5, and TFAP2A for ITGB4 in STAD. Moreover, the functions of ITGB superfamily members were primarily related to regulation of actin cytoskeleton, focal adhesion and PI3K-Akt signalling pathway. The expression of ITGBs was significantly correlated with the infiltration of diverse immune cells, B cell, CD ${ }^{+}$ $\mathrm{T}$ cell, $\mathrm{CD} 4^{+} \mathrm{T}$ cell, macrophage, neutrophil and dendritic cell.

Conclusion: Taken together, our study indicated that ITGB superfamily members could be a potential prognostic target in the development of anti-STAD therapeutics. However, further experimental studies are still necessary to elucidate the mechanism of ITGBs.

\section{Introduction}

Gastric cancer (GC) is the fifth most common malignancy and it is the third leading cause of cancerrelated death, with over 1 million estimated new cases and approximately 800000 deaths in 2018 [1]. GC is a heterogeneous group of tumors with diverse morphologies, molecular models and histogenesis, of which stomach adenocarcinoma (STAD) accounts for 95\% [2]. Since GC still constitutes a global health problem, preventions such as proper diet, early diagnosis and follow-up proper treatments lead to reduction of incidence $[3,4]$. However, it remains the fact that the frequent presence of advanced stage of gastric cancer at first diagnosis, which often narrows the options for the surgical treatment, leading to a worse outcome of patients [5]. Therefore, there is a urgent need to discover the prognostic candidates for gastric cancer's diagnosis and treatment.

Integrin- $\beta$ (ITGB) superfamily, a member of integrin, is made up of eight members in human body, ITGB18 [6]. ITGB superfamily members have been reported to interact with extracellular matrix and activate signal transduction cascades, regulating various cellular processes including proliferation, carcinogenesis and immune responses [7-10]. Zhuang et al reported that ITGB superfamily is closely associated with the prognosis of patients with pancreatic cancer [11]. The correlations between the ITGB 
superfamily members and the prognosis of ovarian cancer were also explored [12]. However, the picture of prognostic and oncologic characteristics of the ITGB superfamily members remains poorly explored in STAD.

In this study, a comprehensive bioinformatics analysis of the mRNA expression of ITGB superfamily members and their potential as biomarkers such as tumor progression, prognosis, gene alterations and immune infiltration in STAD was explored to provide additional data for clinicians to select the appropriate therapeutic schedules, which might lead to a better outcome for patients.

\section{Materials And Methods}

\section{Differentially expressed ITGBs at the transcriptional and protein level}

ONCOMINE (www.oncomine.org) is an integrated online oncogene database and data-mining platform, providing powerful, genemo-wide expression analysis [13]. In this study, a $p$-value $<0.05$, a fold change of 2 and a gene rank in the top $10 \%$ were set as the significant thresholds to explore the aberrant transcriptional levels of ITGBs in different cancer types. The difference in the expression of ITGBs in STAD was analyzed by student's $t$ test.

As a comprehensive, user-friendly and interactive online database, UALCAN (http://ualcan.path.uab.edu/) helps users gather valuable information and data [14]. The expression of ITGBs was analyzed in the "TGCA Gene analysis" module and the "Stomach adenocarcinoma" dataset, in which a statistically significant difference was considered when the $p$ value was $<0.05$.

The HPA database (https://www.proteinatlas.org/) aims to map all the human proteins in cells, tissues and organs, allowing users to conduct the exploration of human proteome [15]. In this study, immunohistochemistry images of protein expression of ITGBs in STAD and normal tissues were directly visualized by HPA.

\section{Associations between the expression of ITGBs and outcomes of STAD patients}

GEPIA (http://gepia.cancer-pku.cn/) is a web server based on TCGA and GTEx data, providing functions such as differential expression analysis, pathological stage analysis, correlation analysis and survival analysis [16]. The pathological stage analysis and survival analysis of ITGBs in STAD, Student's $t$ test was used to generate a $p$ value and the survival analysis was performed using a Kaplan-Meier curve.

Kaplan-Meier Plotter (http://kmplot.com/analysis/) allows users to evaluate the effect of mRNA, miRNA or protein on the survival in breast, ovarian, lung and gastric cancer [17]. The prognostic value of ITGBs in patients with STAD was explored and exhibited together with the HR index, $95 \% \mathrm{Cl}, p$ value and number at risk in the figures. The $p$ value cutoff was 0.05 .

\section{Predicted potential transcription factors (TFs) for prognosis-associated ITGBs in STAD}


NetworkAnalyst (https://www.networkanalyst.ca/) is a comprehensive network visual analytics platform specialized in transcriptome profiling and network analysis for gene expression data [18].

The predicted potential TFs of prognosis-associated ITGBs were explored and visualized as a network picture. TFs regulating two or more prognosis-associated ITGBs were considered as key TFs, and selected to conduct co-expression analysis with their corresponding ITGBs with GEPIA. The differential expression analysis of predicted TFs of tumor and normal tissues were also performed with GEPIA. The threshold of Pearson correlated coefficient $>|0.4|, p$ value $<0.05$. The OS survival analysis of TFs was shown as Kaplan-Meier curves.

\section{Gene alterations and protein-protein interaction (PPI) network of ITGBs}

The genetic alteration of ITGBs in STAD was obtained from cBioPortal (http://www.cbioportal.org/) based on TCGA database, which is an open-access resource for the exploration of multidimensional cancer genomics data sets, allowing users to study the molecular profiles and clinical attributes of cancers [19].

STRING (https://www.string-db.org/) provides physical and functional associated protein-protein interactions, covering more than 24 million and 5 thousand organisms [20]. The PPI network of differentially expressed ITGBs was carried out and 20 relative genes were obtained for further analysis. GeneMANIA (http://genemania.org/) provides quick and convenient access to predicting the function of user-interested genes and gene sets [21]. The functions of differentially expressed ITGBs and associated molecules were explored with GeneMANIA.

\section{Function enrichment analysis of ITGBs}

The Gene Ontology (GO) enrichment and Kyoto Encyclopedia of Genes and Genomes (KEGG) pathway enrichment analysis of ITGB superfamily members and their 20 relative genes were conducted through David 6.8 (https://david.ncifcrf.gov/), a comprehensive, functional annotation online tool helping users understand biological meaning of submitted genes [22]. The top 10 items of each module were visualized as bubble diagrams with Hiplot (https://hiplot.com.cn/), a scientific data analysis and visualization tool. GO enrichment comprises biological process (BP), cellular components (CC) and molecular function (MF).

\section{Immune infiltration analysis of ITGBs}

TIMER (https://cistrome.shinyapps.io/timer/) is comprehensive web tool, providing systematical analysis of immune infiltration of diverse cancer types [23]. The correlations of ITGB superfamily members expression with immune infiltration levels in STAD was performed in "Gene" module, and exhibited as the scatterplot. 


\section{Results}

\section{Aberrant mRNA and protein expressions of ITGB superfamily members in patients with STAD}

ONCOMINE database was utilized in order to research the mRNA expression of ITGB superfamily members in patients with STAD. Compared with normal tissues, the mRNA expressions of ITGB1, ITGB2, ITGB4, ITGB5 and ITGB8 were significantly elevated, while the mRNA expression of ITGB7 was significantly reduced in STAD tissues (FIGURE 1). Data in TABLE 1 was consistent with the results above. For instance, in the Chen dataset the transcriptional levels of ITGB1 were significant higher in gastric intestinal type adenocarcinoma and gastric mixed adenocarcinoma, with the fold changes of 2.130 and 3.145, respectively [24]. In the DErrico dataset, the transcriptional level of ITGB2 was significantly upregulated in diffused gastric adenocarcinoma with the fold change of 2.318 [25]. In the Cho dataset, the transcriptional levels of ITGB7 were significantly reduced in gastric mixed adenocarcinoma, diffused gastric adenocarcinoma and gastric adenocarcinoma with the fold changes of $-5.811,-5.450$ and -4.872 , respectively [26].

Table 1 Transcriptional levels of ITGB superfamily members in different types of gastric cancers and normal tissues (ONCOMINE) 


\begin{tabular}{|c|c|c|c|c|c|}
\hline & Types & Fold Change & P-value & t-test & Reference \\
\hline \multirow[t]{5}{*}{ ITGB1 } & Gastric Intestinal Adenocarcinoma & 2.130 & 8.56E-17 & 10.951 & Chen \\
\hline & Gastric Mixed Adenocarcinoma & 3.145 & $4.08 \mathrm{E}-4$ & 5.242 & Chen \\
\hline & Diffused Gastric Adenocarcinoma & 2.123 & $9.32 \mathrm{E}-4$ & 4.241 & DErrico \\
\hline & Gastric Intestinal Adenocarcinoma & 2.028 & $5.05 \mathrm{E}-4$ & 3.570 & Cho \\
\hline & Gastric Mixed Adenocarcinoma & 2.789 & $9.79 \mathrm{E}-4$ & 3.731 & Cho \\
\hline \multirow[t]{3}{*}{ ITGB2 } & Gastric Mixed Adenocarcinoma & 2.398 & $5.20 \mathrm{E}-4$ & 4.235 & Chen \\
\hline & Diffused Gastric Adenocarcinoma & 2.033 & $4.45 \mathrm{E}-4$ & 3.845 & Chen \\
\hline & Diffused Gastric Adenocarcinoma & 2.318 & 0.003 & 3.624 & DErrico \\
\hline \multirow[t]{2}{*}{ ITGB4 } & Gastric Intestinal Adenocarcinoma & 2.105 & $1.41 \mathrm{E}-4$ & 4.038 & Cho \\
\hline & Gastric Mixed Adenocarcinoma & 2.008 & 0.003 & 3.242 & Cho \\
\hline ITGB5 & Gastric Intestinal Adenocarcinoma & 3.241 & $4.89 \mathrm{E}-9$ & 6.783 & DErrico \\
\hline \multirow[t]{4}{*}{ ITGB7 } & Gastric Mixed Adenocarcinoma & -2.723 & $2.08 \mathrm{E}-5$ & -5.811 & Cho \\
\hline & Diffused Gastric Adenocarcinoma & -2.157 & $8.74 \mathrm{E}-7$ & -5.450 & Cho \\
\hline & Gastric Adenocarcinoma & -2.449 & 0.003 & -4.872 & Cho \\
\hline & Gastric Intestinal Adenocarcinoma & -2.318 & $1.48 \mathrm{E}-4$ & -3.981 & DErrico \\
\hline \multirow[t]{3}{*}{ ITGB8 } & Gastric Mixed Adenocarcinoma & 5.245 & $6.93 \mathrm{E}-5$ & 7.495 & DErrico \\
\hline & Gastric Intestinal Adenocarcinoma & 3.133 & $3.88 \mathrm{E}-9$ & 6.845 & DErrico \\
\hline & Gastric Mixed Adenocarcinoma & 2.092 & $7.71 \mathrm{E}-4$ & 4.403 & Chen \\
\hline
\end{tabular}

Next, the mRNA expression patterns of ITGB superfamily members were measured by the UALCAN database. The results showed that the mRNA expressions of ITGB1 $(p=1.78 \mathrm{e}-04)$, ITGB2 $(p=1.06 \mathrm{E}-$

10), ITGB4 ( $p=1.63 \mathrm{E}-12)$, ITGB5 ( $p=1.67 \mathrm{E}-09)$, ITGB6 $(p=6.17 \mathrm{E}-08)$ and ITGB8 $(p=1.64 \mathrm{E}-12)$ were significantly elevated in STAD vs normal tissues (FIGURE 2). These results were almost consistent with those from ONCOMINE.

After analyzing the mRNA expression of ITGB superfamily members in STAD, the protein expression of ITGB superfamily members were further explored with the Human Protein Atlas. We found that the protein levels of ITGB4, ITGB5, ITGB6 and ITGB8 were significantly up-regulated in STAD tissues (FIGURE 3).

Finally, the correlation between the expressions of ITGB superfamily members and the pathological stage of STAD patients were explored with GEPIA database. As shown in FIGURE 4, there was a significant correlation between the pathological stages and the expression of ITGB2 $(p=0.0143)$ and ITGB7 ( $p=$ 
0.0375). These data suggested that ITGB2 and ITGB7 might play a significant role in the tumorigenesis and progression of STAD.

\section{Prognostic value of ITGB superfamily members in patients with STAD}

The prognostic value of ITGB superfamily members in STAD patients including overall survival (OS) and disease-free survival (DFS) was investigated with GEPIA. The curves in FIGURE 5A suggested that patients with low transcriptional levels of ITGB1 $(p=0.029)$ and ITGB3 $(p=0.039)$ were significantly associated with longer OS. And high transcriptional level of ITGB4 $(p=0.038)$ was associated with longer OS. The results in FIGURE 5B showed that patients with low transcriptional level of ITGB6 $(p=0.011)$ were associated with longer DFS.

The Kaplan-Meier Plotter database was also utilized to analyze the correlation between the mRNA expressions of the ITGB superfamily members and the OS of patients with STAD. As shown in the FIGURE 6 , the patients with low transcriptional levels of ITGB1 $(p=0.01), \operatorname{ITGB} 3(p=0.0076)$ and ITGB5 $(p=$ $0.0022)$ were significantly related to longer OS while a high transcriptional level of ITGB4 $(p=0.045)$ was significantly related to longer OS.

\section{Prediction of TFs for prognosis-associated ITGB superfamily members}

Since the ITGB1, ITGB3, ITGB4, ITGB5 and ITGB6 were significantly associated with the prognosis of patients with STAD, we conducted exploration on the potential TFs for them with NetworkAnalyst database. The visualized network was shown in FIGURE 7A. ITGB1 expression was predicted to be potentially regulated by SP1, CUX1, FHL2, TFAP2A and TFAP2C. ITGB3 expression might be potentially regulated by DAB2 and HOXD3. ITGB4 expression might be potentially regulated by TFAP2A and TFAP2C. DAB2, FHL2 and TFAP2C were predicted to regulate ITGB5. And SP1, CUX1 and FHL2 might potentially regulated ITGB6. Further analysis in FIGURE 7B suggested that the expression of ITGB1 was positively correlated with the expressions of SP1, CUX1 and FHL2, ITGB4 expression was positively associated with TFAP2A expression, and DAB2 expression was positively correlated with the expressions of ITGB3 and ITGB5 (Cor > 0.4, $p<0.05$ ) (GEPIA). However, there was no TF expression positively correlated with ITGB6 expression (FIGURE 7B) (GEPIA). Among the five TFs, the expressions of SP1, CUX1, DAB2 and TFAP2A were significantly up-regulated in STAD tissues vs normal tissues (FIGURE 7C) (GEPIA). Finally, the OS curves by Kaplan-Meier Plotter demonstrated that overexpression of DBA2 was significantly associated with shorter OS while overexpression of TFAP2A was significantly related to longer OS (FIGURE 7D). Therefore, out study implied that DAB2 might synchronously promote the transcription of ITGB3 and ITGB5, and TFAP2A might promote the expression of ITGB4.

\section{Genetic alteration, expression and PPI network of ITGB superfamily members in patients with STAD}

The genetic alterations of ITGB superfamily members in patients with STAD were analyzed by cBioPortal online tool. As a result, ITGB1, ITGB2, ITGB3, ITGB4, ITGB5, ITGB6, ITGB7 and ITGB8 were altered in 9, 8, 
$7,11,8,7,8$ and $11 \%$ of the queried STAD samples, respectively (FIGURE $8 A$ ). Low mRNA expression was the most common alteration in these samples (FIGURE 8B).

Then the PPI network analysis of ITGB superfamily members was conducted to explore the potential interactions among them by STRING. There were 13 nodes and 68 edges in the PPI network (FIGURE 8C). The ITGB superfamily members were associated with pathways such as regulation of actin cytoskeleton and arrhythmogenic right ventricular cardiomyopathy. The GeneMANIA was also utilized to reveal the functions of ITGB superfamily members and their relative molecules (such as WIF1, ATRNL1, EGFL7, TNC, TNR and TENM4), primarily associated with extracellular matrix organization, extracellular structure organization, receptor complex and integrin complex (FIGURE 8D).

\section{Functional enrichment analysis of ITGB superfamily members in patients with STAD}

Firstly, the 20 genes related to ITGB superfamily members were obtained from STRING. Then the functional enrichment analysis of these 28 genes was conducted by DAVID 6.8 and visualized by Hiplot tool. From the FIGURE 9A, the top 10 most highly enriched GO items in the BP category were integrinmediated signaling pathway, extracellular matrix organization, leukocyte migration, cell-matrix adhesion, cell adhesion, heterotypic cell-cell adhesion, cell adhesion mediated by integrin, viral entry into host cell, leukocyte cell-cell adhesion and endodermal cell differentiation, which were significantly regulated by ITGBs in STAD. The integrin complex, cell surface, plasma membrane, focal adhesion, receptor complex, extracellular exosome, external side of plasma membrane, ruffle membrane, cell-cell adherens junction and hemidesmosome were the top 10 most highly enriched items in the CC category (FIGURE 9B). In the MF category, the ITGB superfamily members and their relative genes were mainly enriched in integrin binding, receptor activity and virus receptor activity (FIURE 9C).

As shown in FIGURE 9D, the top 10 KEGG pathways affected by ITGBs in STAD were regulation of actin cytoskeleton, focal adhesion, hypertrophic cardiomyopathy, dilated cardiomyopathy, ECM-receptor interaction, arrhythmogenic right ventricular cardiomyopathy, PI3K-Akt signaling pathway, proteoglycans in cancer, cell adhesion molecules and pathways in cancer.

\section{Immune cell infiltration of ITGB superfamily members in patients with STAD}

The proliferation and progression of cancer cell is associated with immune cell levels. Therefore, the associations between ITGB superfamily members and immune cell infiltration were explored by TIMER database (FIGURE 10). The expression of ITGB1 was positively associated with the infiltration of CD4 ${ }^{+} \mathrm{T}$ cell, macrophage and dendritic cell. The expressions of ITGB2 and ITGB3 were both significantly positively related to the infiltration levels of all six immune cells, B cell, $\mathrm{CD} 8^{+} \mathrm{T}$ cell, $\mathrm{CD} 4^{+} \mathrm{T}$ cell, macrophage, neutrophil and dendritic cell. There was a remarkably negative correlation between the expression of ITGB4 and the infiltration of $\mathrm{CD} 8^{+} \mathrm{T}$ cell, $\mathrm{CD} 4^{+} \mathrm{T}$ cell and dendritic cell. ITGB5 expression was positively associated with the infiltration of $\mathrm{CD} 4^{+} \mathrm{T}$ cell, macrophage and dendritic cell, and negatively associated with the infiltration of $B$ cell. We also found the higher the infiltration levels of $B$ cell, the higher the expression of ITGB6. The expression of ITGB7 was significantly positively related to 
the infiltration of $\mathrm{CD}^{+}{ }^{+} \mathrm{T}$ cell, $\mathrm{CD} 4^{+} \mathrm{T}$ cell, macrophage, neutrophil and dendritic cell. ITGB8 expression was positively associated with the infiltration of B cell.

\section{Discussion}

Gastric cancer displays marked molecular heterogeneity with aggressive behavior and treatment resistance, exhibiting a high mortality rate with an average 5 -year survival rate of $20 \%$ in most areas of the world $[27,28]$. The exploration of potential prognostic biomarkers is necessary for improving the bad outcomes for patients with STAD.

Integrins- $\beta$ (TIGB) superfamily, made up of ITGB1, ITGB2, ITGB3, ITGB4, ITGB5, ITGB6, ITGB7 and ITGB8, is a member of integrin, which is a kind of cell cohesion protein, playing an important role in cell migration, hemostasis and immune response [29]. Besides, integrin takes part in mediating the initiation, progression and metastasis of tumor [30,31]. ITGB1 overexpression can promote the progression of prostate cancer through upregulating the expression of caveolin-1 [32]. ITGB2 enhances the glycolysis activity through PI3K/AKT/mTOR pathways, which plays a significant role in cancer associated fibroblasts in the promotion of oral squamous cell carcinoma [33]. It has been reported that ITGB3dependent uptake of extracellular vesicles, for which focal adhesion kinase is required as a key role, could promote the metastasis of breast cancer cells [34]. ITGB4 and ITGB6 targeted immunotherapy is a promising strategy, showing a great effect on the inhibition of the progression and metastasis of tumors $[35,36]$. Studies have also suggested that ITGB7 and ITGB8 have the potential to mediate the progression of tumors, paving the way for a novel therapeutic approach for the treatment for cancers [37-39].

In this study, the mRNA expression of ITGB superfamily members in STAD was firstly explored. The transcriptional levels of ITGB1, ITGB2, ITGB4, ITGB5, ITGB6 and ITGB8 were significantly up-regulated and the transcriptional level of ITGB7 was significantly down-regulated in the STAD tissues compared with normal tissues. Besides, through analyzing the protein expression of ITGB superfamily members in STAD by HPA, we found that the protein expressions of ITGB4, ITGB5, ITGB6 and ITGB8 were higher in STAD tissues than normal tissues. Next, results suggested that the expression of ITGB2 and ITGB7 increased as the tumors progressed. STAD patients with low expression of ITGB1, ITGB3 and ITGB5 and high expression of ITGB4 were significantly associated with better overall survival. And the low expression of ITGB6 in STAD patients was related to better disease-free survival. These data demonstrate that the differentially expressed ITGB superfamily members may play a significant role in the tumorigenesis, progression and prognosis of STAD.

TF functions in the transcriptional level as key regulators in the gene regulation [40]. Our study suggests that ITGB1, ITGB3, ITGB4, ITGB5 and ITGB6 may serve as potential prognostic biomarkers for STAD. Therefore, the predicted TFs for the prognosis-associated ITGBs were explored based on the Network Analysis database. Results identified DAB2 as the potential TFs for ITGB3 and ITGB5, and TFAP2A as the potential TF for ITGB4. Both of DAB2 and TFAP2A were obviously up-regulated in STAD. The overexpression of DAB2 was significantly associated with shorter OS, consistent with the results that 
overexpression of ITGB3 and ITGB5 was related to shorter OS. And the same conclusion can be found that overexpression of TFAP2A and ITGB4 was significantly associated with longer OS. Therefore, we supposed that DAB2 might enhance the expression of ITGB3 and ITGB5, and TFAP2A might enhance the expression of ITGB4. Our study may provide deeper insight into the potential mechanisms of ITGB3, ITGB4 and ITGB6 in gastric adenocarcinoma.

As well as the differential expression of genes in STAD, gene mutations and epigenetic alterations play a non-negligible role in the progression of tumors [41]. There were frequent genetic alterations in the ITGB superfamily members in STAD, where the low mRNA expression was the most alteration. The gene alteration analysis may also help to understand the role of ITGBs in STAD.

Then the function of ITGB superfamily members was assessed using GO enrichment analysis and KEGG pathway enrichment analysis. As expected, the functions of ITGBs and relative 20 genes are mainly related to regulation of actin cytoskeleton, focal adhesion and PI3K-Akt signaling pathway. Actin cytoskeleton is formed by polymerization of globular actin monomers to form microfilaments, and participated in several essential cellular processes including endocytosis, intracellular transport, cell morphogenesis and cell motility $[42,43]$. Moreover, the regulation of actin cytoskeleton plays a significant role in biological processes associated with the invasion and metastasis of tumors [44, 45]. Focal adhesion is reported to promote the progression of cancers when activated by follistatin-like protein [46]. The key role of PI3K-Akt signaling pathway in the tumorigenesis, proliferation and progression in cancers has also been proven $[47,48]$. These data indicate that ITGB superfamily members could be potential drug therapeutic targets.

Integrins could mediate the migration and location of immune cells $[49,50]$. More and more evidence suggested that immune cell infiltration is the key role affecting the progression and recurrence of the tumor [51]. In our study, the expression of ITGB superfamily members was significantly associated with the infiltration of six immune cells, $\mathrm{B}$ cells, $\mathrm{CD} 8^{+} \mathrm{T}$ cells, $\mathrm{CD} 4^{+} \mathrm{T}$ cells, macrophages, neutrophils and dendritic cells, indicating that ITGBs could affect the prognosis of tumors through mediating the immune status.

There were some limitations in our study. The analysis involved in the study were all based on different online databases. And further studies consisting of cell experiments and clinical studies are required to validate the results and explore the potential mechanisms underlying the roles of ITGB superfamily members in STAD.

\section{Conclusion}

In conclusion, our study indicated that differentially expressed ITGBs could be potential prognostic biomarkers for the outcomes of patients with STAD, might providing novel insights for the design of new anti-STAD therapeutics. 


\section{Abbreviations}

ITGB: Integrin $\beta$; GC: Gastric cancer; STAD: Stomach adenocarcinoma; OS: Overall survival; DFS: Diseasefree survival; TGCA: The cancer genome atlas; TF: Transcription factor; PPI: Protein-protein interaction; GO: Gene ontology; KEGG: Kyoto Encyclopedia of Genes and Genomes; BP: Biological process; CC: Cellular components; MF: Molecular function; ECM: Extracellular matrix; PI3K: Phosphatidylinositol 3 kinase; mTOR: mammalian target of rapamycin.

\section{Declarations}

\section{Ethics approval and consent to participate}

Not applicable.

\section{Consent for publication}

Not applicable.

\section{Availability of data and materials}

All data generated or analysed during this study are included in this published article.

\section{Competing interests}

The authors declare that they have no competing interests.

\section{Funding}

None.

\section{Authors' contributions}

All authors participated in the manuscript. Peixin Lu and Jinying Jiang: study design, bioinformatics analysis and writing the manuscript. Jun Lu: critical revision of the manuscript.

\section{Acknowledgements}

We would like to thank the database used above and their contributors for their help and support.

\section{References}

1. Bray F, Ferlay J, Soerjomataram I, Siegel RL, Torre LA, Jemal A. Global cancer statistics 2018: GLOBOCAN estimates of incidence and mortality worldwide for 36 cancers in 185 countries. CA Cancer J Clin. 2018;68(6):394-424. 
2. Yakirevich E, Resnick MB. Pathology of gastric cancer and its precursor lesions. Gastroenterol Clin North Am. 2013;42(2):261-84.

3. Gao JP, Xu W, Liu WT, Yan M, Zhu ZG. Tumor heterogeneity of gastric cancer: From the perspective of tumor-initiating cell. World J Gastroenterol. 2018;24(24):2567-81.

4. Park JY, von Karsa L, Herrero R. Prevention strategies for gastric cancer: a global perspective. Clin Endosc. 2014;47(6):478-89.

5. Thrumurthy SG, Chaudry MA, Chau I, Allum W. Does surgery have a role in managing incurable gastric cancer? Nature Reviews Clinical Oncology. 2015;12(11):676-82.

6. Hynes RO. Integrins: versatility, modulation, and signaling in cell adhesion. Cell. 1992;69(1):11-25.

7. Shattil SJ, Kim C, Ginsberg MH. The final steps of integrin activation: the end game. Nat Rev Mol Cell Biol. 2010;11(4):288-300.

8. Hynes RO. Integrins: bidirectional, allosteric signaling machines. Cell. 2002;110(6):673-87.

9. LaFlamme SE, Mathew-Steiner S, Singh N, Colello-Borges D, Nieves B. Integrin and microtubule crosstalk in the regulation of cellular processes. Cell Mol Life Sci. 2018;75(22):4177-85.

10. Bianconi D, Unseld M, Prager GW. Integrins in the Spotlight of Cancer. Int J Mol Sci. 2016;17(12).

11. Zhuang H, Zhou Z, Ma Z, Li Z, Liu C, Huang S, et al. Characterization of the prognostic and oncologic values of ITGB superfamily members in pancreatic cancer. J Cell Mol Med. 2020;24(22):13481-93.

12. Zhu T, Chen R, Wang J, Yue H, Lu X, Li J. The prognostic value of ITGA and ITGB superfamily members in patients with high grade serous ovarian cancer. Cancer Cell Int. 2020;20:257.

13. Rhodes DR, Kalyana-Sundaram S, Mahavisno V, Varambally R, Yu J, Briggs BB, et al. Oncomine 3.0: genes, pathways, and networks in a collection of 18,000 cancer gene expression profiles. Neoplasia. 2007;9(2):166-80.

14. Chandrashekar DS, Bashel B, Balasubramanya SAH, Creighton CJ, Ponce-Rodriguez I, Chakravarthi B, et al. UALCAN: A Portal for Facilitating Tumor Subgroup Gene Expression and Survival Analyses. Neoplasia. 2017;19(8):649-58.

15. Thul PJ, Akesson L, Wiking M, Mahdessian D, Geladaki A, Ait Blal H, et al. A subcellular map of the human proteome. Science. 2017;356(6340).

16. Tang Z, Li C, Kang B, Gao G, Li C, Zhang Z. GEPIA: a web server for cancer and normal gene expression profiling and interactive analyses. Nucleic Acids Res. 2017;45(W1):W98-102.

17. Nagy A, Munkacsy G, Gyorffy B. Pancancer survival analysis of cancer hallmark genes. Sci Rep. 2021;11(1):6047.

18. Zhou G, Soufan O, Ewald J, Hancock REW, Basu N, Xia J. NetworkAnalyst 3.0: a visual analytics platform for comprehensive gene expression profiling and meta-analysis. Nucleic Acids Res. 2019;47(W1):W234-W41.

19. Gao J, Aksoy BA, Dogrusoz U, Dresdner G, Gross B, Sumer So, et al. Integrative analysis of complex cancer genomics and clinical profiles using the cBioPortal. Sci Signal. 2013;6(269):pl1. 
20. Szklarczyk D, Gable AL, Lyon D, Junge A, Wyder S, Huerta-Cepas J, et al. STRING v11: protein-protein association networks with increased coverage, supporting functional discovery in genome-wide experimental datasets. Nucleic Acids Res. 2019;47(D1):D607-D13.

21. Warde-Farley D, Donaldson SL, Comes O, Zuberi K, Badrawi R, Chao P, et al. The GeneMANIA prediction server: biological network integration for gene prioritization and predicting gene function. Nucleic Acids Res. 2010;38(Web Server issue):W214-20.

22. Huang da W, Sherman BT, Lempicki RA. Systematic and integrative analysis of large gene lists using DAVID bioinformatics resources. Nat Protoc. 2009;4(1):44-57.

23. Li T, Fan J, Wang B, Traugh N, Chen Q, Liu JS, et al. TIMER: A Web Server for Comprehensive Analysis of Tumor-Infiltrating Immune Cells. Cancer Res. 2017;77(21):e108-e10.

24. Chen X, Leung SY, Yuen ST, Chu KM, Ji J, Li R, et al. Variation in gene expression patterns in human gastric cancers. Mol Biol Cell. 2003;14(8):3208-15.

25. D'Errico M, de Rinaldis E, Blasi MF, Viti V, Falchetti M, Calcagnile A, et al. Genome-wide expression profile of sporadic gastric cancers with microsatellite instability. Eur J Cancer. 2009;45(3):461-9.

26. Cho JY, Lim JY, Cheong JH, Park YY, Yoon SL, Kim SM, et al. Gene expression signature-based prognostic risk score in gastric cancer. Clin Cancer Res. 2011;17(7):1850-7.

27. Yan HHN, Siu HC, Law S, Ho SL, Yue SSK, Tsui WY, et al. A Comprehensive Human Gastric Cancer Organoid Biobank Captures Tumor Subtype Heterogeneity and Enables Therapeutic Screening. Cell Stem Cell. 2018;23(6):882-97. e11.

28. Karimi P, Islami F, Anandasabapathy S, Freedman ND, Kamangar F. Gastric cancer: descriptive epidemiology, risk factors, screening, and prevention. Cancer Epidemiol Biomarkers Prev. 2014;23(5):700-13.

29. Gahmberg CG, Gronholm M, Madhavan S, Jahan F, Mikkola E, Viazmina L, et al. Regulation of cell adhesion: a collaborative effort of integrins, their ligands, cytoplasmic actors, and phosphorylation. Q Rev Biophys. 2019;52:e10.

30. Desgrosellier JS, Cheresh DA. Integrins in cancer: biological implications and therapeutic opportunities. Nat Rev Cancer. 2010;10(1):9-22.

31. Hamidi H, Ivaska J. Every step of the way: integrins in cancer progression and metastasis. Nat Rev Cancer. 2018;18(9):533-48.

32. Pellinen T, Blom S, Sanchez S, Valimaki K, Mpindi JP, Azegrouz H, et al. ITGB1-dependent upregulation of Caveolin-1 switches TGFbeta signalling from tumour-suppressive to oncogenic in prostate cancer. Sci Rep. 2018;8(1):2338.

33. Zhang $X$, Dong $Y$, Zhao M, Ding L, Yang X, Jing Y, et al. ITGB2-mediated metabolic switch in CAFs promotes OSCC proliferation by oxidation of NADH in mitochondrial oxidative phosphorylation system. Theranostics. 2020;10(26):12044-59.

34. Fuentes P, Sese M, Guijarro PJ, Emperador M, Sanchez-Redondo S, Peinado H, et al. ITGB3-mediated uptake of small extracellular vesicles facilitates intercellular communication in breast cancer cells. Nat Commun. 2020;11(1):4261. 
35. Ruan S, Lin M, Zhu Y, Lum L, Thakur A, Jin R, et al. Integrin beta4-Targeted Cancer Immunotherapies Inhibit Tumor Growth and Decrease Metastasis. Cancer Res. 2020;80(4):771-83.

36. Liang B, Shahbaz M, Wang Y, Gao H, Fang R, Niu Z, et al. Integrinbeta6-targeted immunoliposomes mediate tumor-specific drug delivery and enhance therapeutic efficacy in colon carcinoma. Clin Cancer Res. 2015;21(5):1183-95.

37. Neri P, Ren L, Azab AK, Brentnall M, Gratton K, Klimowicz AC, et al. Integrin beta7-mediated regulation of multiple myeloma cell adhesion, migration, and invasion. Blood. 2011;117(23):6202-13.

38. Mertens-Walker I, Fernandini BC, Maharaj MS, Rockstroh A, Nelson CC, Herington AC, et al. The tumour-promoting receptor tyrosine kinase, EphB4, regulates expression of integrin-beta8 in prostate cancer cells. Bmc Cancer. 2015;15:164.

39. Malric L, Monferran S, Delmas C, Arnauduc F, Dahan P, Boyrie S, et al. Inhibiting Integrin beta8 to Differentiate and Radiosensitize Glioblastoma-Initiating Cells. Mol Cancer Res. 2019;17(2):384-97.

40. Anguita E, Candel FJ, Chaparro A, Roldan-Etcheverry JJ. Transcription Factor GFI1B in Health and Disease. Front Oncol. 2017;7:54.

41. Chia NY, Tan P. Molecular classification of gastric cancer. Ann Oncol. 2016;27(5):763-9.

42. Papakonstanti EA, Stournaras C. Cell responses regulated by early reorganization of actin cytoskeleton. Febs Lett. 2008;582(14):2120-7.

43. Morris HT, Machesky LM. Actin cytoskeletal control during epithelial to mesenchymal transition: focus on the pancreas and intestinal tract. Br J Cancer. 2015;112(4):613-20.

44. Peng JM, Bera R, Chiou CY, Yu MC, Chen TC, Chen CW, et al. Actin cytoskeleton remodeling drives epithelial-mesenchymal transition for hepatoma invasion and metastasis in mice. Hepatology. 2018;67(6):2226-43.

45. Ye YP, Jiao HL, Wang SY, Xiao ZY, Zhang D, Qiu JF, et al. Hypermethylation of DMTN promotes the metastasis of colorectal cancer cells by regulating the actin cytoskeleton through Rac1 signaling activation. J Exp Clin Cancer Res. 2018;37(1):299.

46. Gu C, Wang X, Long T, Wang X, Zhong Y, Ma Y, et al. FSTL1 interacts with VIM and promotes colorectal cancer metastasis via activating the focal adhesion signalling pathway. Cell Death Dis. 2018;9(6):654.

47. Ediriweera MK, Tennekoon KH, Samarakoon SR. Role of the PI3K/AKT/mTOR signaling pathway in ovarian cancer: Biological and therapeutic significance. Semin Cancer Biol. 2019;59:147-60.

48. Fattahi S, Amjadi-Moheb F, Tabaripour R, Ashrafi GH, Akhavan-Niaki H. PI3K/AKT/mTOR signaling in gastric cancer: Epigenetics and beyond. Life Sci. 2020;262:118513.

49. Park EJ, Peixoto A, Imai Y, Goodarzi A, Cheng G, Carman CV, et al. Distinct roles for LFA-1 affinity regulation during T-cell adhesion, diapedesis, and interstitial migration in lymph nodes. Blood. 2010;115(8):1572-81.

50. Svensson L, Stanley P, Willenbrock F, Hogg N. The Galphaq/11 proteins contribute to T lymphocyte migration by promoting turnover of integrin LFA-1 through recycling. Plos One. 2012;7(6):e38517. 
51. Bindea G, Mlecnik B, Tosolini M, Kirilovsky A, Waldner M, Obenauf AC, et al. Spatiotemporal dynamics of intratumoral immune cells reveal the immune landscape in human cancer. Immunity. 2013;39(4):782-95.

\section{Figures}

\begin{tabular}{|c|c|c|c|c|c|c|c|c|c|c|c|c|c|c|c|c|}
\hline \multirow{2}{*}{$\begin{array}{l}\text { Analysis Type by Cancer } \\
\text { Bladder Cancer }\end{array}$} & \multicolumn{2}{|c|}{\begin{tabular}{|c|}
$\begin{array}{c}\text { Cancer } \\
\text { vs. } \\
\text { Normal }\end{array}$ \\
ITGB1
\end{tabular}} & \multicolumn{2}{|c|}{$\begin{array}{l}\begin{array}{c}\text { Cancer } \\
\text { vs. } \\
\text { Normal }\end{array} \\
\text { ITGB2 }\end{array}$} & \multicolumn{2}{|c|}{\begin{tabular}{|c}
$\begin{array}{c}\text { Cancer } \\
\text { vs. } \\
\text { Normal }\end{array}$ \\
ITGB3
\end{tabular}} & \multicolumn{2}{|c|}{\begin{tabular}{|c|}
$\begin{array}{c}\text { Cancer } \\
\text { vs. } \\
\text { Normal }\end{array}$ \\
ITGB4
\end{tabular}} & \multicolumn{2}{|c|}{\begin{tabular}{|c}
$\begin{array}{c}\text { Cancer } \\
\text { vs. } \\
\text { Normal }\end{array}$ \\
ITGB5
\end{tabular}} & \multicolumn{2}{|c|}{$\begin{array}{c}\begin{array}{c}\text { Cancer } \\
\text { vs. } \\
\text { Normal }\end{array} \\
\text { ITGB6 }\end{array}$} & \multicolumn{2}{|c|}{$\begin{array}{l}\begin{array}{c}\text { Cancer } \\
\text { vs. } \\
\text { Normal }\end{array} \\
\text { ITGB7 }\end{array}$} & \multicolumn{2}{|c|}{$\begin{array}{l}\text { Cancer } \\
\text { vs. } \\
\text { Normal } \\
\text { ITGB8 }\end{array}$} \\
\hline & & 1 & 1 & 1 & & 1 & 3 & & & & & & 1 & & & \\
\hline Brain and CNS Cancer & 9 & 1 & 9 & & 3 & & 5 & & 8 & 1 & & 2 & & 1 & 4 & 1 \\
\hline Breast Cancer & & 4 & 7 & 1 & & 4 & & 2 & 1 & 1 & 1 & 3 & 2 & 1 & 1 & 4 \\
\hline Cervical Cancer & 1 & & & & & & 3 & & & & 1 & & & & & \\
\hline Colorectal Cancer & 2 & & & 1 & & & 5 & & & & & 4 & & 2 & 7 & \\
\hline Esophageal Cancer & & 2 & 1 & & 1 & & 5 & & & & & & & & & 2 \\
\hline Gastric Cancer & 5 & 1 & 4 & & & & 2 & & 1 & & & & & 4 & 4 & \\
\hline Head and Neck Cancer & 4 & & 2 & & & 1 & 7 & & 2 & & 6 & & & & 1 & \\
\hline Kidney Cancer & 3 & 6 & 8 & & 6 & 4 & 4 & 2 & & & & 3 & & & 1 & 4 \\
\hline Leukemia & 4 & & 2 & 9 & & 2 & & & 1 & & 1 & 2 & 6 & & & \\
\hline Liver Cancer & & & 1 & & & & & & & & & & & & 1 & 1 \\
\hline Lung Cancer & & & & 4 & & & 6 & 2 & & & & 5 & & & 2 & \\
\hline Lymphoma & 2 & 3 & 4 & 2 & & & & 3 & 7 & & 1 & 2 & 1 & 1 & & 3 \\
\hline Melanoma & 1 & & 1 & & & & 1 & 2 & & 2 & & 1 & & & & 1 \\
\hline Myeloma & & 2 & & 3 & & & & & & 1 & & & & & & \\
\hline Other Cancer & 5 & & 4 & & 2 & 1 & 2 & 2 & 6 & & 2 & 3 & 2 & & 1 & 3 \\
\hline Ovarian Cancer & & 1 & & & & & 1 & & & 1 & 3 & & 1 & & 2 & \\
\hline Pancreatic Cancer & 2 & & 3 & & & 2 & 4 & 1 & 5 & & 3 & 2 & & & 1 & \\
\hline Prostate Cancer & 1 & 1 & & 1 & & 2 & 1 & 3 & 1 & & & 3 & & & 2 & 2 \\
\hline Sarcoma & 6 & & & 2 & 3 & & & 7 & & 5 & 2 & 2 & & 1 & 1 & \\
\hline Significant Unique Analyses & 43 & 22 & 47 & 24 & 14 & 17 & 49 & 22 & 32 & 11 & 19 & 31 & 13 & 10 & 28 & 21 \\
\hline Total Unique Analyses & 4. & & & & & & & & & & & & 43 & & & \\
\hline
\end{tabular}

\section{Figure 1}

The mRNA expression of ITGB superfamily members in gastric cancers (ONCOMINE). The figure shows the numbers of datasets with statistically alterations in the mRNA expression of ITGBs: up-regulated (red) and down-regulated (blue). The transcriptional levels of ITGB1, 2, 4, 5 and 8 were significantly elevated and the transcriptional level of ITGB7 was significantly reduced in gastric cancer vs normal tissues. 

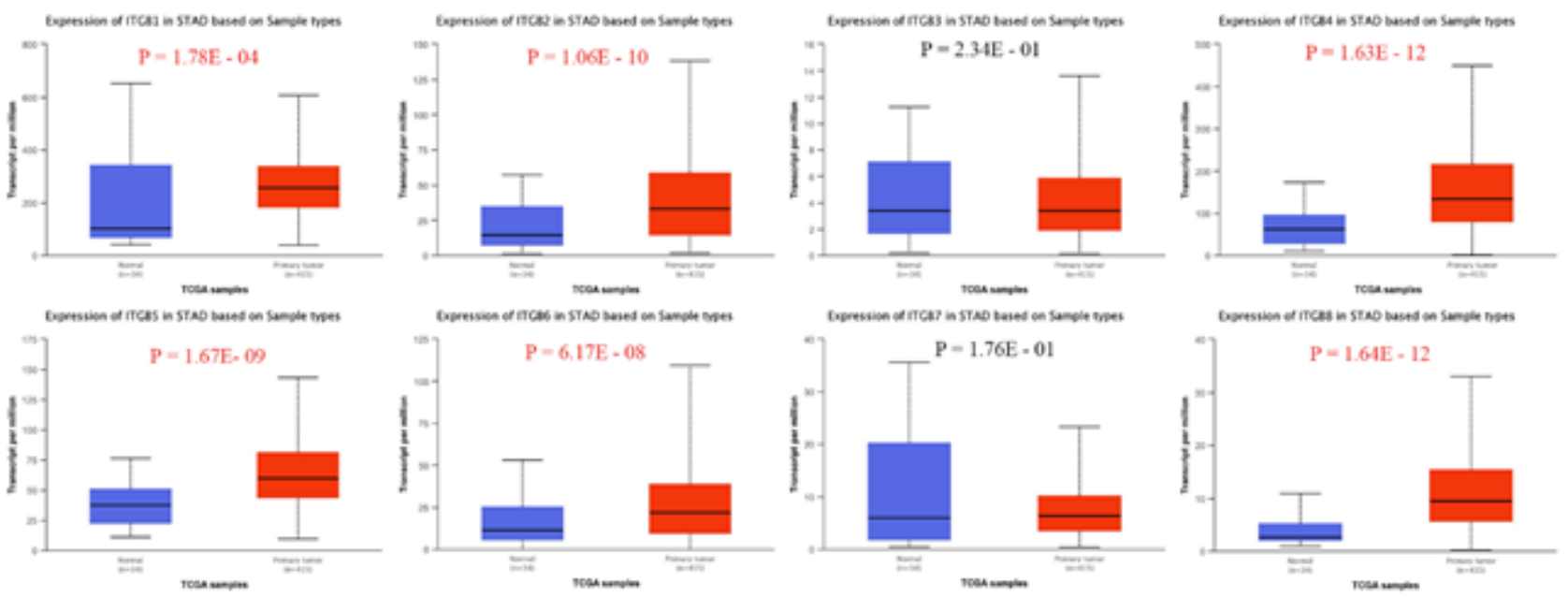

Figure 2

The expression of ITGB superfamily members in STAD (UALCAN). The expression of ITGB1, 2, 4, 5, 6 and 8 was significantly elevated in STAD tissues compared with normal tissues $(p<0.05)$.
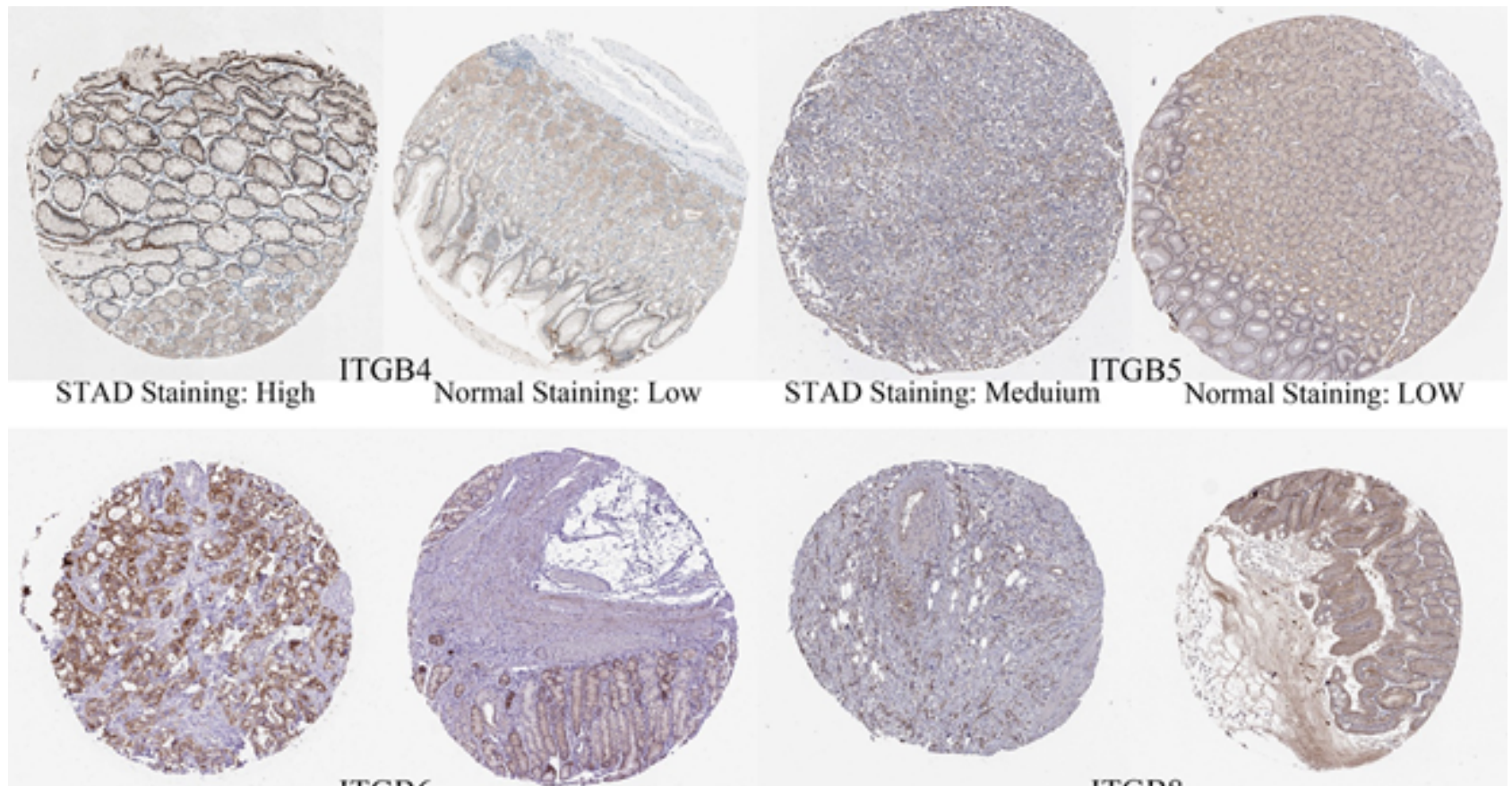

STAD Staining: Medium

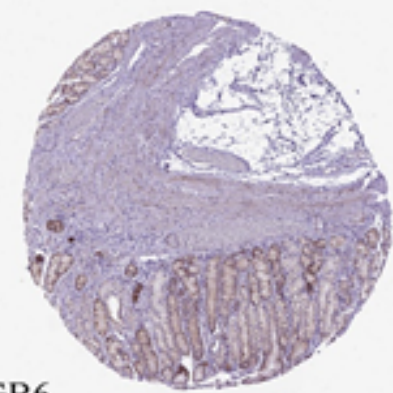

Normal Staining: Low

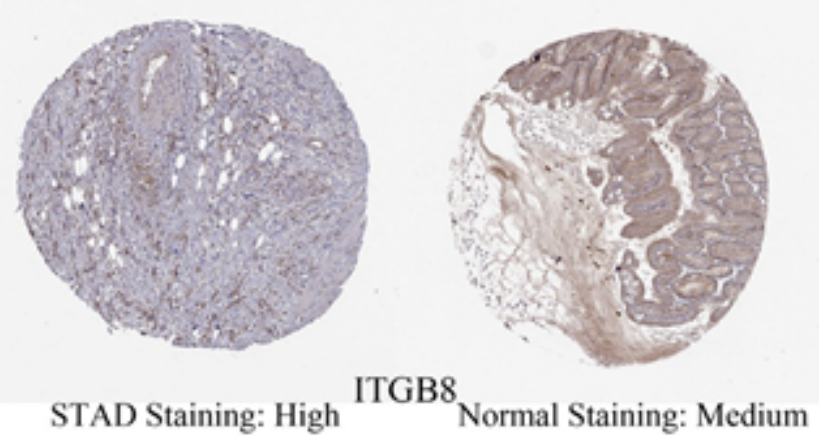

STAD Staining: High ${ }^{1 T G B}$ Normal Staining: Medium

\section{Figure 3}

The protein expression of ITGB superfamily members in STAD vs normal tissues (HPA). The protein expression of ITGB4, 5, 6 and 8 was higher in STAD than normal tissues.
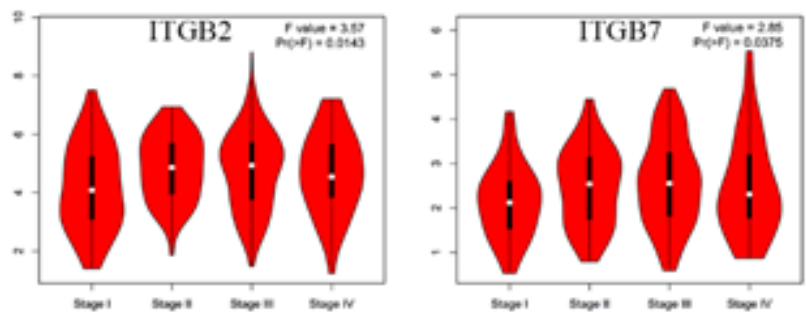


\section{Figure 4}

The correlations between the pathological stage of STAD and the expression of ITGB2 and ITGB7 (GEPIA) $(p<0.05)$.
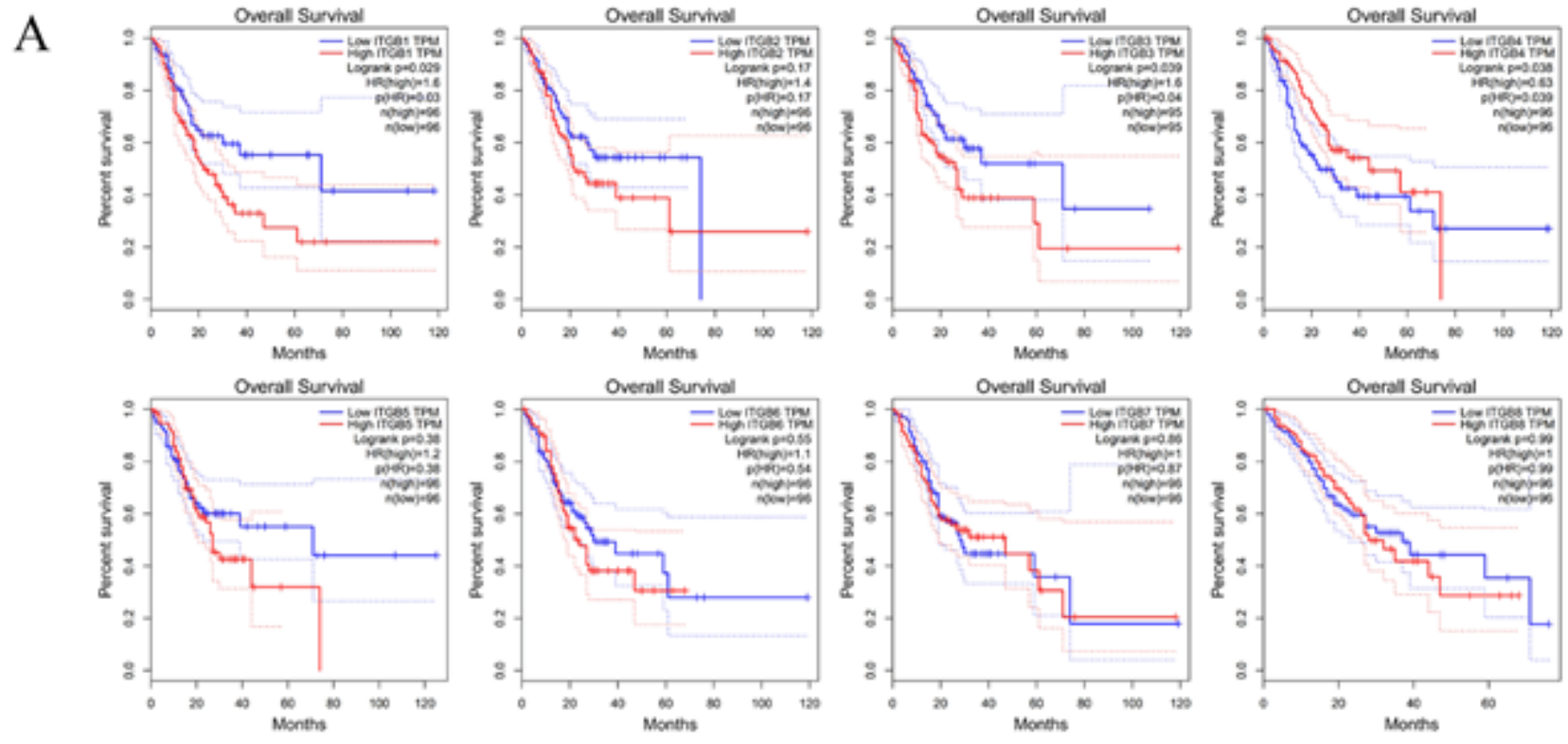

B
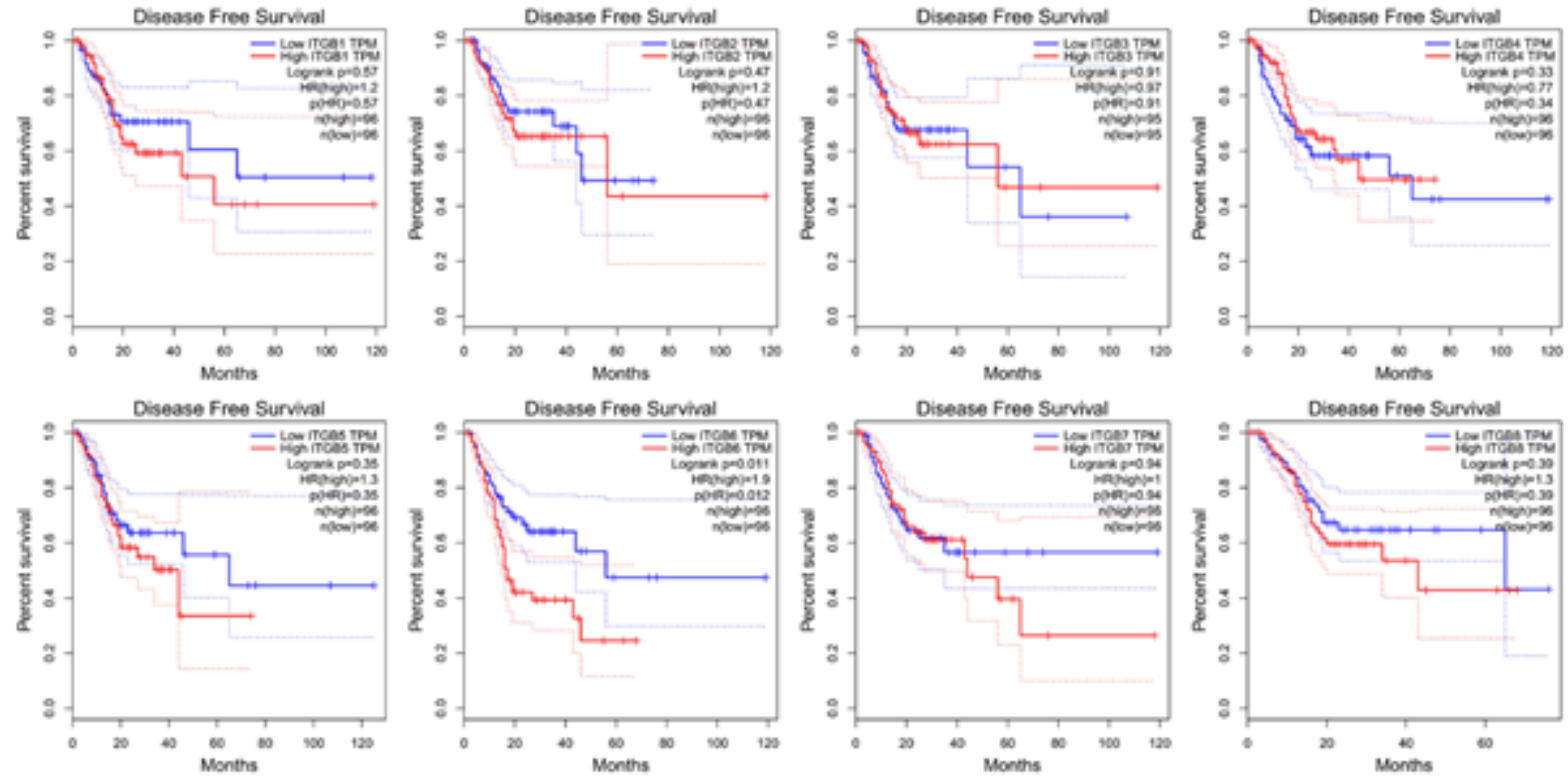

\section{Figure 5}

The prognostic value of ITGB superfamily members in STAD (GEPIA). (A) The OS curves of ITGBs. Low transcriptional levels of ITGB1 $(p=0.029)$ and ITGB3 $(p=0.039)$ were significantly related to longer OS and high transcriptional level of ITGB4 ( $p=0.038)$ was significantly related to longer OS; (B) The DFS curves of ITGBs. Patients with a low expression of ITGB6 $(p=0.011)$ were significantly associated with longer DFS. 

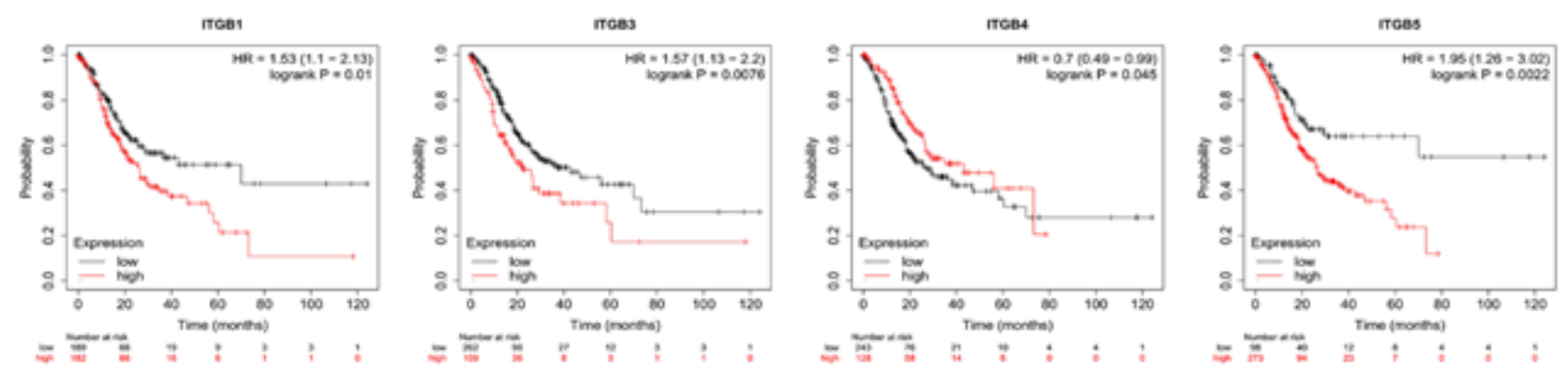

\section{Figure 6}

The prognostic value of ITGB superfamily members in STAD (Kaplan-Meier Plotter). High expression of ITGB1 $(P=0.01)$, ITGB3 $(p=0.0078)$ and ITGB5 $(p=0.0022)$ was significantly associated with shorter OS in STAD patients. And low expression of ITGB4 $(p=0.045)$ was significantly associated with shorter OS in STAD patients.
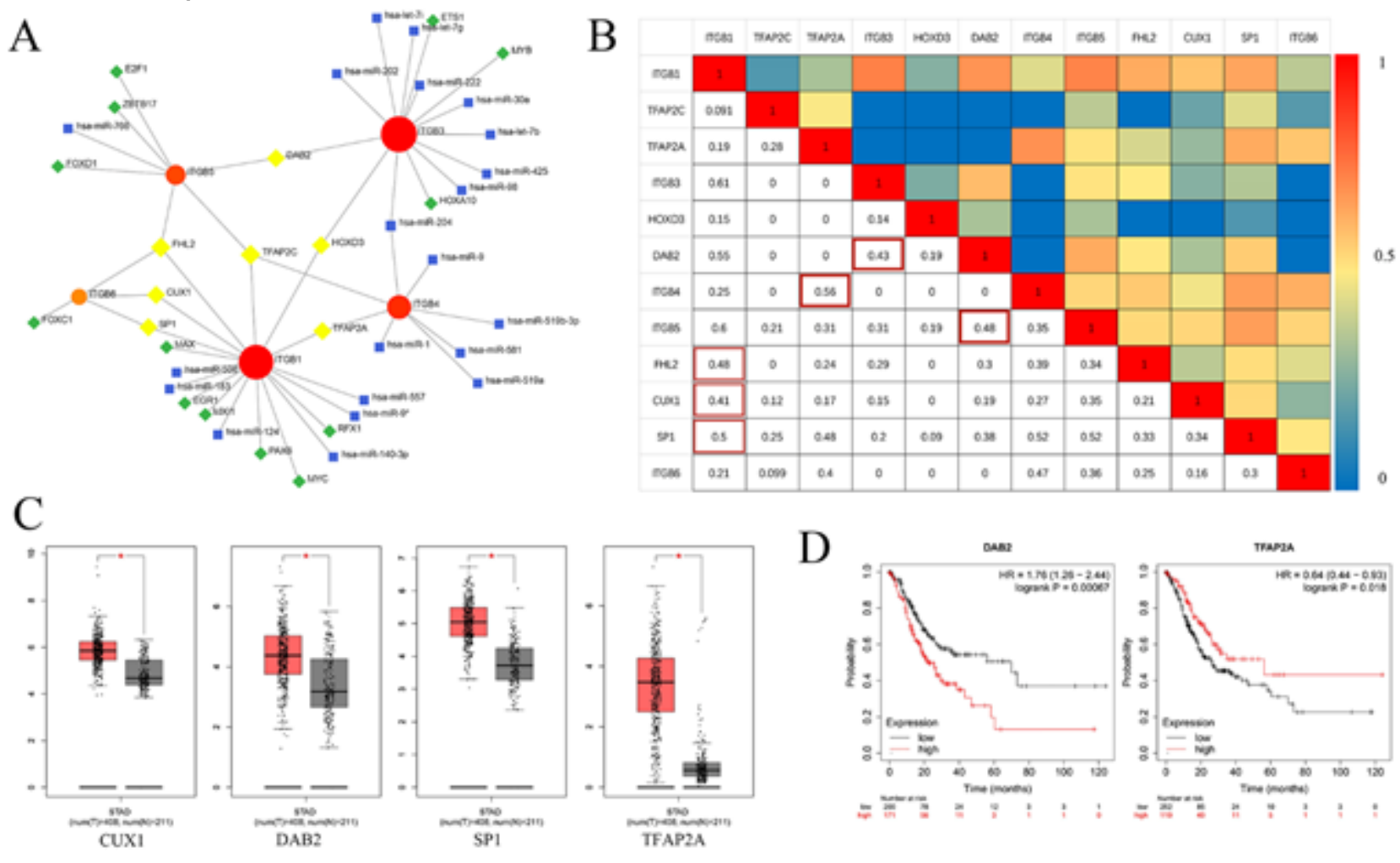

Figure 7

The potential transcription factors (TF) for prognosis-associated ITGBs in STAD. (A) The TF-miRNA coregulation network of ITGB1, 3, 4, 5 and 6 (NetworkAnalyst). SP1, CUX1, FHL2, DAB2, HOXD3, TFAP2A and TFAP2C were key TFs regulating 2 or more ITGBs (yellow). Green: TFs only regulating one kind of ITGBs. Blue: miRNA; (B) The correlations of ITGB1, 3, 4, 5 and 6, and their corresponding TFs; (C) CUX1, DAB2, SP1 and TFAP2A were significantly up-regulated in STAD $(p<0.05)$ (GEPIA); (D) DAB2 overexpression was significantly associated with worse OS and low expression of TFAP2A was significantly associated with worse OS (Kaplan-Meier Plotter). 
A

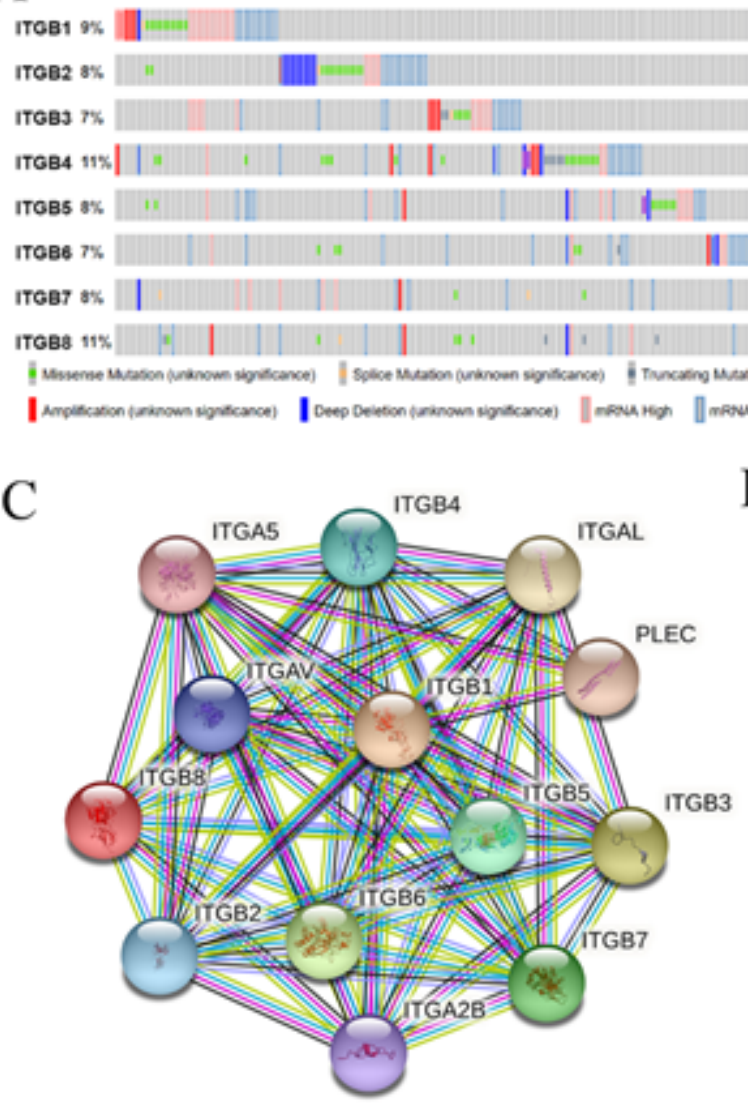

B

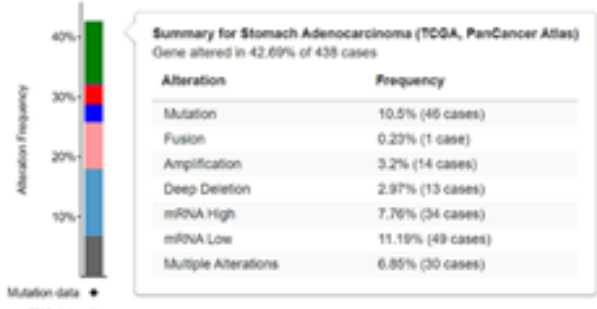

nowisas.

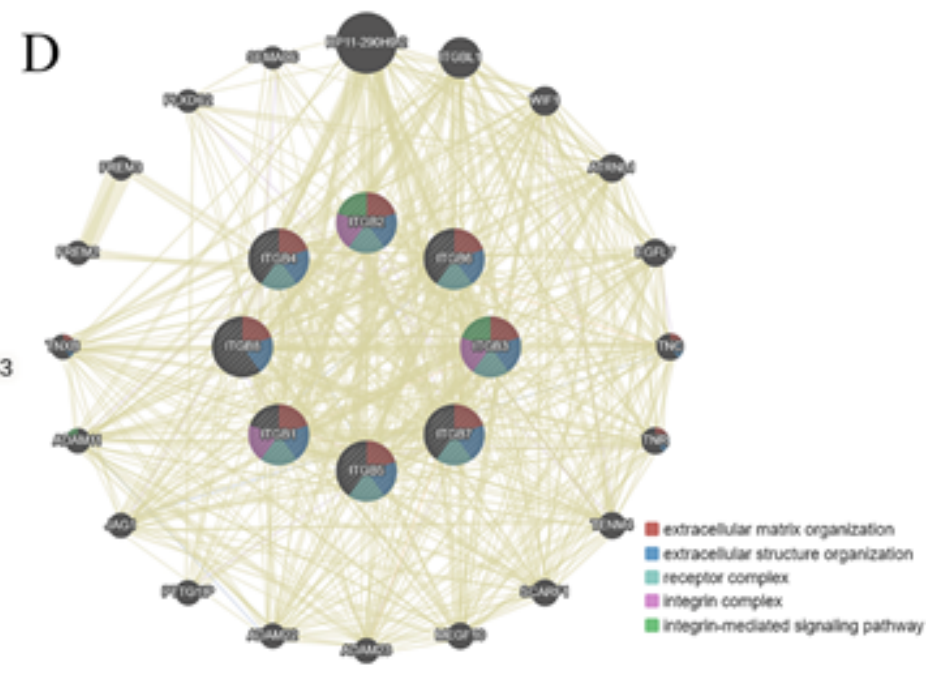

Figure 8

Genetic alterations and protein-protein interaction network of ITGB superfamily members (A, B) Genetic alterations of ITGBs in STAD. Low mRNA was the most common change (cBioPortal); (C, D) The proteinprotein interaction networks of ITGBs (STRING and GeneMANIA).

A

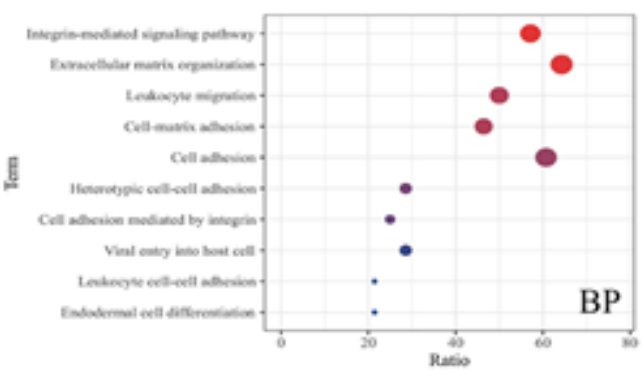

$\mathrm{C}$

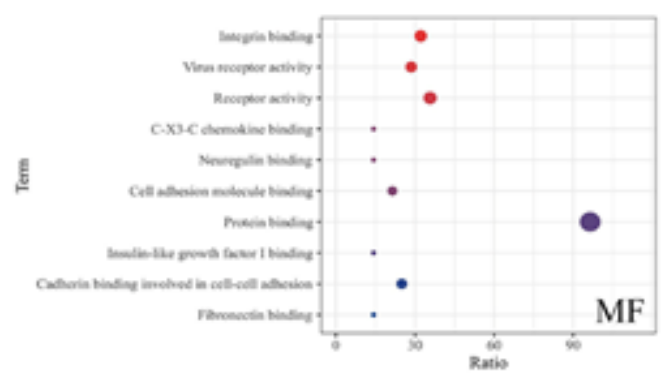

B

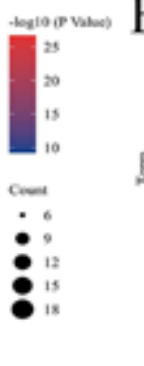

D

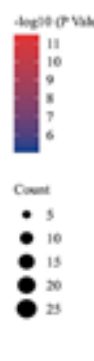

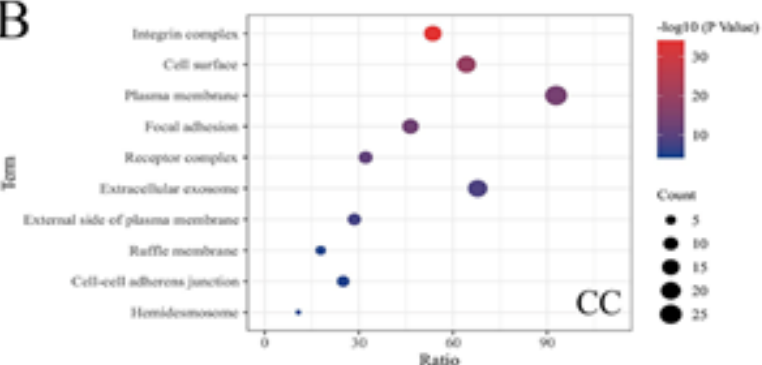

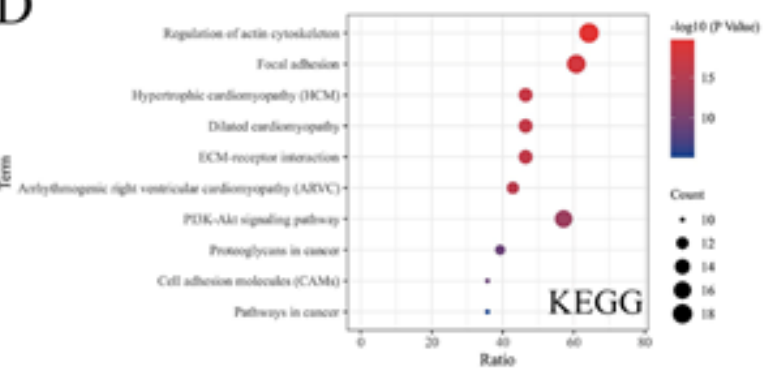

Figure 9 
The GO and KEGG pathway enrichment analysis of ITGB superfamily members and their 20 relative genes (David 6.8). (A-C) Bubble plots of GO enrichment in BP terms, CC terms and MF terms; (D) Bubble plot of KEGG pathway enrichment terms.
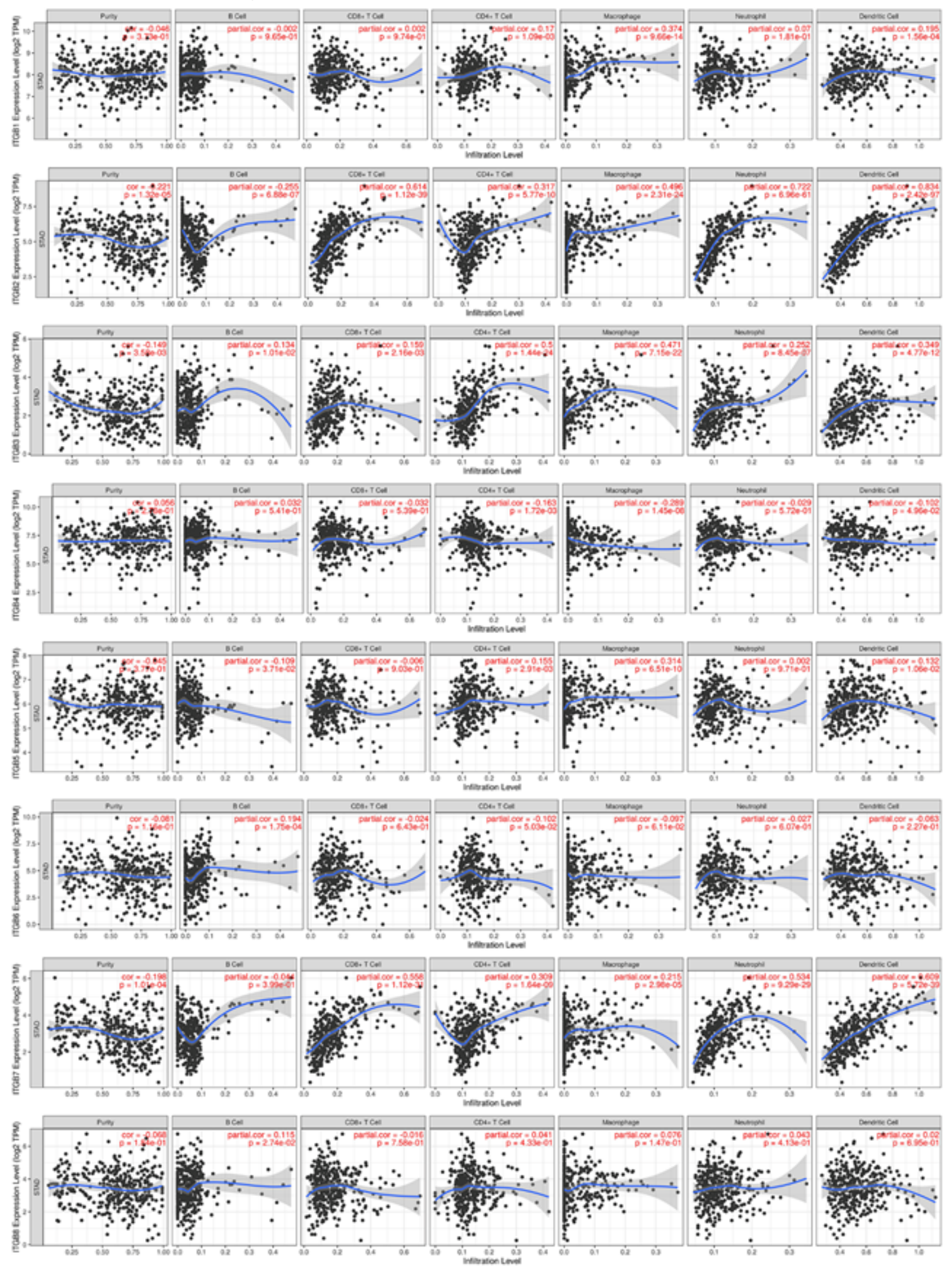

Figure 10

Correlations between ITGB superfamily members and the infiltration of immune cells in STAD (TIMER). The mRNA expression of ITGB superfamily members was significantly related to the immune infiltration 
levels in STAD.

Page 21/21 